\title{
A 3D trabecular bone homogenization technique
}

\author{
M. MARQUES ${ }^{1,2 *}$, J. BELINHA ${ }^{1,3}$, A.F. OLIVEIRA ${ }^{4}$, \\ M.C. MANZANARES CESPEDES ${ }^{5}$, R.M. NATAL JORGE ${ }^{1,2}$ \\ ${ }^{1}$ Institute of Science and Innovation in Mechanical and Industrial Engineering, Porto, Portugal. \\ ${ }^{2}$ Faculty of Engineering of University of Porto, Porto, Portugal. \\ ${ }^{3}$ Department of Mechanical Engineering, School of Engineering, Polytechnic of Porto, Porto, Portugal. \\ ${ }^{4}$ Institute of Biomedical Sciences Abel Salazar, Porto, Portugal. \\ ${ }^{5}$ Muscular and Skeletal Pathology Research, Human Anatomy and Embryology Unit, \\ Universitat de Barcelona, Barcelona, Spain.
}

\begin{abstract}
Purpose: Bone is a hierarchical material that can be characterized from the microscale to macroscale. Multiscale models make it possible to study bone remodeling, inducing bone adaptation by using information of bone multiple scales. This work proposes a computationally efficient homogenization methodology useful for multiscale analysis. This technique is capable to define the homogenized microscale mechanical properties of the trabecular bone highly heterogeneous medium. Methods: In this work, a morphology-based fabric tensor and a set of anisotropic phenomenological laws for bone tissue was used, in order to define the bone micro-scale mechanical properties. To validate the developed methodology, several examples were performed in order to analyze its numerical behavior. Thus, trabecular bone and fabricated benchmarks patches (representing special cases of trabecular bone morphologies) were analyzed under compression. Results: The results show that the developed technique is robust and capable to provide a consistent material homogenization, indicating that the homogeneous models were capable to accurately reproduce the micro-scale patch mechanical behavior. Conclusions: The developed method has shown to be robust, computationally less demanding and enabling the authors to obtain close results when comparing the heterogeneous models with equivalent homogenized models. Therefore, it is capable to accurately predict the micro-scale patch mechanical behavior in a fraction of the time required by classic homogenization techniques.
\end{abstract}

Key words: fabric tensor, homogenization technique, multiscale; bone

\section{Introduction}

Bone is a complex mineralized biological structure defined by bone matrix and by bone cells. It can be classified as a hierarchical material possessing distinct scales from the nanoscale to the macroscale [21]. To comprehend bone physiology, it is necessary to understand bone at its different scales. Therefore, bone is studied at its macroscale level, [14], microscale level [20], and also crossing scale levels (multiscale analyses) [22]. Bone remodeling is one of the most trend research topics in computational biomechanics. In this process, bone cells are responsible to renew the bone matrix, by creating or reabsorbing it [25]. This process is progressive and it is triggered to allow for bone adaptation to any new external loads. As bone is a hierarchical structure, its morphology adaptation leads to changes of its macro-scale mechanical properties, needed to fulfill and optimize the bone-specific physiological function of structural support [7]. Bone remodeling can be studied using a multi-scale approach, where information from two or more scales can be used to define bone different functional requirements at different scales, reflecting bone's different behavior at different scales [13]. Wolff [26] reported that the directions of the external applied loads directly influence the direction of the trabecular bone. As a consequence, changes

\footnotetext{
* Corresponding author: Marco Costa Marques, Institute of Science and Innovation in Mechanical and Industrial Engineering, Campus da FEUP Rua Dr. Roberto Frias, 400, 4200-465, Porto, Portugal. Phone: +351 229578 710, e-mail: mcmarques@inegi.up.pt

Received: April 2nd, 2020

Accepted for publication: July 6th, 2020
} 
in bone's trabecular structure (at a microscale level) influence its macroscale mechanical behavior, which, in turn, impacts the remodeling process.

Today, it is common to use computational biomechanics to study this type of biological problems [2]. The numerical methods and discretization techniques used in computational biomechanics enable the researchers to define, approximate and mimic biological processes using mathematical descriptions of the problem. Bio-mathematical formulations combined with distinct discretization approaches, such as finite element methods (FEM) [27] and meshless methods [2], make it possible to describe the mechanical behavior of bone at certain scale levels by using multiscale approaches [15] and homogenization techniques [19]. As in many other subjects, the use of the numerical approaches to study bone remodeling began with simplistic models. At first, it was considered that bone was only sensitive to mechanical stimuli [6]. In early studies, bone tissues started to be considered as an isotropic-elastic material, a consideration that disregard the importance of material orientation and structure in the remodeling process. In the last few years, the complexity of bone models gradually increased, and models started to be combined with multiscale methods [15], in which homogenization techniques are required [23]. Trabecular bone is characterized by having a highly heterogeneous morphology, possessing a high surface-area-volume ratio. Thus, trabecular bone is the major responsible for bone's metabolic activity, including the remodeling process. Thus, considering that many of bone remodeling studies are performed using discretization methods, the highly heterogeneous trabecular bone domain can have a negative impact on the performance of these methods. Hence, some authors started to use homogenization techniques which allowed them do define highly heterogeneous domain as a homogeneous domain of the trabecular bone, but considering its orientation and anisotropy [17]. Homogenization techniques are defined using a variety of approaches. In the literature, numerous examples of homogenization techniques application are found, with different approaches to define bone mechanical properties. The majority of the works use micro-CT images as a source for representing bone structure, [10], [16]-[19], but other authors use different methods, such as scanning acoustic microscopy (SAM) [5]. The method to define the mechanical properties of the homogeneous domain usually relies on the definition of a tensor that enables the researchers to relate the bone microstructure with bone mechanical properties. Imaging-based approaches, such as gray-level structure tensor (GST) or Mean Intercept
Length (MIL) method, are the most often used to acquire these tensors [12], [16]-[18], although there are other approaches, such as using Fast Fourier Transform (FFT) homogenization methods [5]. These homogenization techniques make it possible to determine equivalent mechanical properties of a heterogeneous material by substituting the volume of the heterogeneous material with an equivalent volume of homogeneous material so as to predict the behavior of the macro-scale using the mechanical properties coming from the micro-scale. The fabric tensor concept, A, a symmetric second rank tensor, enables the researcher to characterize the arrangement of a multiphase material. Back in 1985, Cowin [8] described the relation between the fabric tensor and the fourth rank elasticity tensor $\mathbf{C}_{i j k l}$. The fabric tensor also provides information that allows to define an ellipsoid that can be related with the several material symmetries observed in many natural materials, such as bone tissue. The fabric tensor can be defined using two different methods, a mechanically-based method and the morphologybased method. The MIL method [24], a morphologybased method that uses the interface between phases of the material to estimate the tensor, is the most used technique to compute a fabric tensor [16]. When considering the bone structure, it is possible to obtain information regarding the material phase-change using computed tomography scan images, leading, naturally, to the use of morphology-based techniques to define the fabric tensor. The morphology-based methods use an orientation distribution function (ODF), which is estimated from an orientation-dependent feature of interest. ODF disposes the data in a polar plot and, then, fits the data with an ellipse. As Cowin initially suggested [8], it was afterwards demonstrated in the literature that the ODF fitted ellipse (specifically, the ellipse parameters) can be correlated with the material orientation, i.e., the material anisotropy [24]. The fabric tensor concept makes it possible to numerically define bone mechanical proprieties, which is of high importance when studying the structural behavior of bone tissue or its biological processes, such as bone remodeling. Defining the mechanical properties enables the researchers to feed constitute laws, in order to better simulate the structural behavior of bone tissue. With this, the main objective of this work was to develop a new 3D homogenization technique, by applying the fabric tensor concept combined with a previously developed bone tissue material law and with FEM, aiming to achieve a low-cost and efficient multiscale technique. The novelty of this homogenization technique, that enables us to mechanically define bone microstructure, is the combination of the fabric tensor 
concept with a previously defined bone tissue material law, acquired using experimental data.

\section{Materials and methods}

The objective of this work was to develop a homogenization technique based on the fabric tensor concept. Thus, a 3D MIL method was used to define an ellipsoid encoding the material anisotropy and orientation. This 3D MIL method uses a micro-CT bone image Representative Volume Elements (RVE) as input, which enables it to represent the bone structure. The methodology was evaluated using a set of RVE benchmarks. Having the ellipsoid parameters and using a phenomenological material law according to Belinha et al. [4] and Agic et al. [1], it was possible to define an homogenized domain representative of a highly heterogeneous trabecular domain. Then, a set of mechanical simulations were performed, in which heterogeneous models were used. The geometry of these heterogeneous models was defined by micro-CT images of the representative trabecular bone. Additionally, homogenous geometrical models with regular shape (cubes) were constructed, with their mechanical proprieties defined using the developed homogenization technique.

\subsubsection{MIL}

To define the fabric tensor encoding bone anisotropy, the method requires a medical image as input. These images are usually a set of grey-scale images, thus, the first operation of this method is to segment the medical images using the Otsu method. This enables us to define in the RVE which voxels are bone material or void. The second step of the method is defined by an iterative process, where a random search using a sphere centered within the RVE boundaries is selected. This search sphere contains a set of vectors with random directions that are set constant in all the iterations. In each iteration, the search sphere centroid changes, and the intersection between the vectors from the vector list and the bone voxels are counted. These intersections are only counted if the bone voxel centroid has a maximum distance from the vector of at least the voxel size. As the vector list is constant within the iterative process, it is possible to sum the number of bone voxels that are intercepted in all the iterations for each vector. In this work, depending on the benchmark, the iterative process has a minimum and a maximum of 1000 and 5000 iterations, respectively, and has a variance stop criteria of 0.08 . Using equation (1), it is possible to transform the intersection count into Cartesian coordinates. For each direction, $l$, represented by a unique vector, it is multiplied the number of iterations, iter, with the original vector size, $h$. Dividing this value by the number of interceptions of the vector with direction $\operatorname{Int}(\imath)$ for all the iterations, the $\operatorname{MIL}(l)$ is obtained. The original vector size, $h$, was defined by the search sphere size, that contained the vector list. Having the MIL value, the ODF data, it is possible to fit it into an ellipsoid using Fitzgibbon method. [9] After defining the ellipsoid, relevant parameters for the methodology are extracted. Specifically, the parameters extracted are the radius that define the ellipsoid, $r_{1}, r_{2}$ and $r_{3}$ being $r_{1}>r_{2}>r_{3}$, and the angles theta, $\theta$, and azimuth $\omega$, defined from $r_{1}$, that defined the ellipsoid 3D orientation. $r_{1}, r_{2}$ and $r_{3}$ are normalized by the $r_{1}$ size, being $r_{1}=1$ always.

$$
\operatorname{MIL}(\imath)=\frac{h \times i t e r}{\operatorname{Int}(\imath)} .
$$

\subsection{Material law}

Using the parameters obtained from the 3D MIL method, the RVE image information, and the phenomenological material laws obtained from Belinha et al. [4] and Agic et al. [1], it is possible to create and define a material law.

The segmented image information was used to define the bone apparent density, $\rho_{a p p}$. It was obtained using a relation between the number of white voxels (bone voxels), $w_{v}$, and the black voxels (void voxels), $b_{v}$, as described in Eq. (2). In this equation, $\rho_{O}=2.1 \mathrm{~g} / \mathrm{cm}^{3}$ represents the bone typical maximum apparent density.

$$
\rho_{a p p}=\frac{w_{v}}{\left(b_{v}+w_{v}\right)} \times \rho_{O}\left[\mathrm{~g} / \mathrm{cm}^{3}\right] .
$$

Also, using the image information, through the universal mixture laws, was possible to define $v_{1}$, Eq. (3), being $v=0.3$ the typical Poisson ratio for cortical bone (bone voxels, at the microscale).

$$
v_{1}=\frac{\left(0 \times b_{v}\right)+\left(0.3 \times w_{v}\right)}{\left(b_{v}+w_{v}\right)} .
$$

Regarding $v_{2}$ and $v_{3}$, in this work, the values obtained from Agic et al. [1] are assumed (Fig. 1), where a relation between $v_{2}$ and $v_{3}$ and the bone ap- 


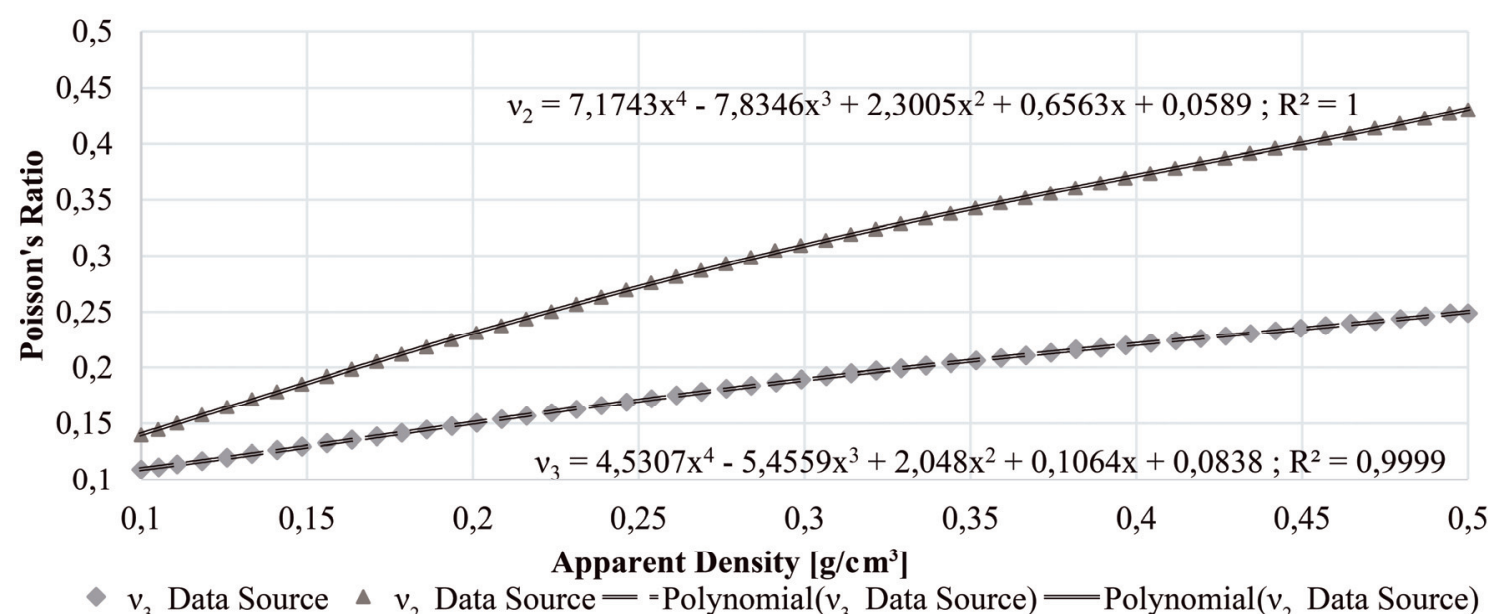

Fig. 1. Data obtained from Agic et al. [1], fitted to polynomial functions

parent density, $\rho_{a p p}$, is shown. Fitting Agic et al. data it was possible to define two fourth order polynomial equations, Eqs. (4) and (5), which enabled us to describe the behavior of $v_{2}$ and $v_{3}$ analytically, considering $\rho_{a p p}$.

$$
\begin{gathered}
v_{2}= \\
\left\{\begin{array}{c}
7.1743 \rho_{a p p}^{4}-7.8346 \rho_{a p p}^{3}+2.3005 \rho_{a p p}^{2}+0.6563 \rho_{a p p} \\
+0.0589 \rightarrow \text { if } \rho_{a p p}<0.5 \mathrm{~g} / \mathrm{cm}^{3} \\
0.43 \rightarrow \text { if } \rho_{a p p}>0.5 \mathrm{~g} / \mathrm{cm}^{3}
\end{array}\right.
\end{gathered}
$$

$$
\begin{gathered}
v_{3}= \\
\left\{\begin{array}{c}
4.5307 \rho_{a p p}^{4}-5.4559 \rho_{a p p}^{3}+2.048 \rho_{a p p}^{2} \\
+0.1064 \rho_{a p p}+0.0838 \rightarrow \text { if } \rho_{a p p}<0.5 \mathrm{~g} / \mathrm{cm} \\
0.245 \rightarrow \text { if } \rho_{a p p}>0.5 \mathrm{~g} / \mathrm{cm}^{3}
\end{array} .\right.
\end{gathered}
$$

Considering Belinha's material law [3] and using Eq. (6) and coefficients from Table 1, it is possible to define $E_{1}$. Concerning $E_{2}$ and $E_{3}$, it is a defined relation between $E_{1}$ and the radius $r_{1}, r_{2}$ and $r_{3}$. Thus, $E_{2}$ is defined using a relation between $r_{1}, r_{2}$ and $E_{1}$ (Eq. (7)), and $E_{3}$, is defined using $r_{1}, r_{3}$ and $E_{1}$, (Eq. (8)).

Table 1. Coefficients obtained from Belinha's material law [3]

\begin{tabular}{|c|c|c|c|c|}
\hline & $j=0$ & $j=1$ & $j=2$ & $j=3$ \\
\hline$a_{j}$ & $0.0 \mathrm{E}+00$ & $7.216 \mathrm{E}+02$ & $8.059 \mathrm{E}+02$ & $0.0 \mathrm{E}+00$ \\
\hline$b_{j}$ & $-1.770 \mathrm{E}+05$ & $3.861 \mathrm{E}+05$ & $-2.798 \mathrm{E}+05$ & $6.836 \mathrm{E}+04$ \\
\hline
\end{tabular}

$$
\begin{gathered}
E_{1}=\left\{\begin{array}{cc}
\sum_{j=3}^{0} a_{j} \rho_{a p p}^{j} & \text { if } \rho_{a p p} \leq 1.3 \mathrm{~g} / \mathrm{cm}^{3} \\
\sum_{j=3}^{0} b_{j} \rho_{a p p}^{j} & \text { if } \rho_{a p p}>1.3 \mathrm{~g} / \mathrm{cm}^{3}
\end{array},\right. \\
E_{2}=\frac{r_{2} \times E_{1}}{r_{1}}, \\
E_{3}=\frac{r_{3} \times E_{1}}{r_{1}} .
\end{gathered}
$$

The shear modulus, $G_{1}, G_{2}$ and $G_{3}$, are approximated using Eqs. (9), (10) and (11), respectively:

$$
\begin{aligned}
& G_{1}=\frac{E_{1}}{2 \times\left(1+v_{1}\right)}, \\
& G_{2}=\frac{E_{2}}{2 \times\left(1+v_{2}\right)}, \\
& G_{3}=\frac{E_{3}}{2 \times\left(1+v_{3}\right)} .
\end{aligned}
$$

Using the proposed method, it is possible to define a set of mechanical properties capable of defining a homogenized $\operatorname{RVE}\left(E_{1}, E_{2}, E_{3}, v_{1}, v_{2}, v_{3}, G_{1}, G_{2}, G_{3}\right.$ and $\theta$ and $\omega$ ). It made it possible to construct a constitutive matrix $\mathbf{c}_{o x^{\prime} y^{\prime} z^{\prime}}$, equation (12) for the $o x^{\prime} y^{\prime} z^{\prime}$ local coordinate system (oriented with the material principal axis, following the principal "axial" and "transverse" directions).Using $\theta$ and $\omega$ obtained from the ellipsoid fitting, the transformation rotation matrices $\mathbf{T}$, are defined (Eqs. (13) and (14)). 


$$
\begin{aligned}
& \mathbf{c}_{o x^{\prime} y^{\prime} z^{\prime}}=\left[\begin{array}{cccccc}
\frac{1}{E_{1}} & \frac{-v_{1}}{E_{2}} & \frac{-v_{3}}{E_{3}} & 0 & 0 & 0 \\
\frac{-v_{1}}{E_{1}} & \frac{1}{E_{2}} & \frac{-v_{2}}{E_{3}} & 0 & 0 & 0 \\
\frac{-v_{3}}{E_{1}} & \frac{-v_{2}}{E_{2}} & \frac{1}{E_{3}} & 0 & 0 & 0 \\
0 & 0 & 0 & \frac{1}{G_{1}} & 0 & 0 \\
0 & 0 & 0 & 0 & \frac{1}{G_{2}} & 0 \\
0 & 0 & 0 & 0 & 0 & \frac{1}{G_{3}}
\end{array}\right], \\
& {\left[\mathbf{T}_{o y}\right]=\left[\begin{array}{cccccc}
\cos ^{2} \omega & 0 & \sin ^{2} \omega & 0 & 0 & \sin 2 \omega \\
0 & 1 & 0 & 0 & 0 & 0 \\
\sin ^{2} \omega & 0 & \cos ^{2} \omega & 0 & 0 & -\sin 2 \omega \\
0 & 0 & 0 & \cos \omega & \sin \omega & 0 \\
0 & 0 & 0 & \sin \omega & \cos \omega & 0 \\
-\sin \beta \cos \omega & 0 & \sin \beta \cos \omega & 0 & 0 & \cos ^{2} \omega-\sin ^{2} \omega
\end{array}\right]} \\
& {\left[\mathbf{T}_{o z}\right]_{\theta}=\left[\begin{array}{cccccc}
\cos ^{2} \theta & \sin ^{2} \theta & 0 & -\sin 2 \theta & 0 & 0 \\
\sin ^{2} \theta & \cos ^{2} \theta & 0 & \sin 2 \theta & 0 & 0 \\
0 & 0 & 1 & 0 & 0 & 0 \\
\sin ^{2} \theta & -\sin ^{2} \theta & 0 & \cos ^{2} \theta-\sin ^{2} \theta & 0 & 0 \\
0 & 0 & 0 & 0 & \cos \theta & -\sin \theta \\
0 & 0 & 0 & 0 & \sin \theta & \cos \theta
\end{array}\right] .}
\end{aligned}
$$

Afterwards, it is possible to define the material constitutive matrix in the global axis with Eq. (15),

$$
\mathbf{c}_{o x y z}=\left[\mathbf{T}_{o z}\right]_{\theta}^{T}\left[\left[\mathbf{T}_{o y}\right]_{\omega}^{T} \mathbf{c}_{o x^{\prime} y^{\prime} z^{\prime}}\left[\mathbf{T}_{o y}\right]_{\omega}\right]\left[\mathbf{T}_{o z}\right]_{\theta} .
$$

\subsection{Algorithm robustness}

In order to evaluate the robustness of the 3D MIL method, benchmark examples were analyzed. One of the factors that could influence the method was the number of vectors in the vector list present inside the search sphere. Thus, the behavior of the method was evaluated assuming distinct vector list sizes with: 50, $100,250,1000$ and 1500 vectors. These sets are represented in Fig. 2. Furthermore, 6 benchmarks RVEs were fabricated with well-defined geometric characteristics, containing bone-voxels and void-voxels, being bone voxels represented in blue (Fig. 3). Benchmark 1 (Fig. 3a), has a well-defined vertical material principal direction. Benchmark 2 (Fig. 3b), has exactly the same number of bone and void voxels as benchmark 1, but it was obtained applying a rotation in order to have a horizontal material principal direction. Benchmark 3 (Fig. 3c), and benchmark 4 (Fig. 3d) have an oblique material principal direction. The difference between benchmarks 3 and 4, is the number of voxels that define the thickness of the bone region. Benchmark 5 (Fig. 3e) and benchmark 6 (Fig. 3f) have the same material principal direction, but different number of bone voxels defining the bone voxels central column. The corresponding ellipsoids and material directions are shown in Figs. $3 \mathrm{~g}-1$. It was expected that the material first principal direction was collinear with the material distribution. With Figures $3 \mathrm{~g}-1$, it is possible to observe that using the proposed technique, the obtained material directions are in accordance with the expected material directions of each benchmark. The study of these benchmarks aimed to evaluate the obtained mechanical proprieties, prior to the transformation 


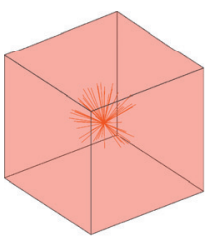

(a)

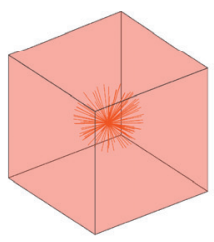

(b)

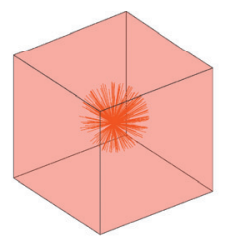

(c)

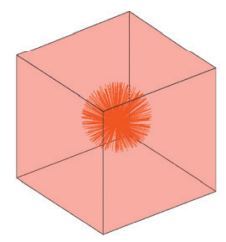

(d)

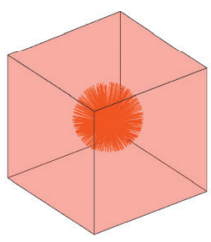

(e)

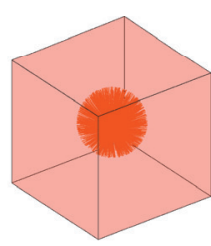

(f)

Fig. 2. Set of image is representing different numbers of vectors inside the search sphere: (a) 50 vectors, (b) 100 vectors, (c) 250 vectors, (d) 500 vectors, (e) 1000 vectors, (f) 1500 vectors

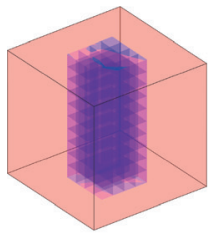

(a)

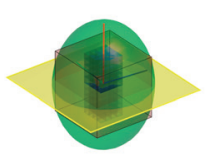

(g)

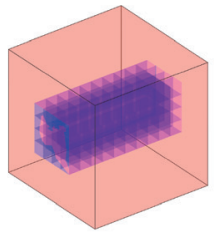

(b)

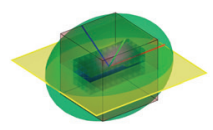

(h)

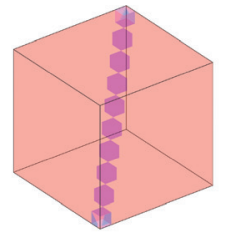

(c)

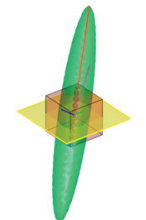

(i)

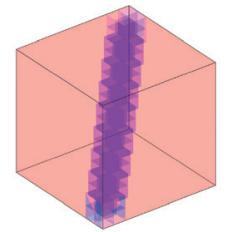

(d)

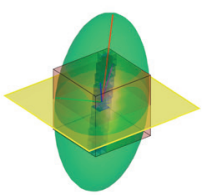

(j)

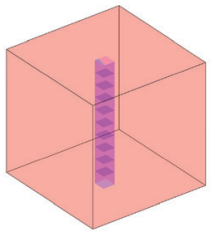

(e)

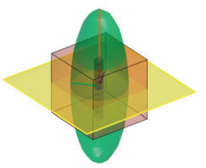

(k)

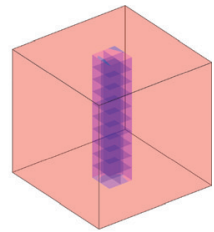

(f)

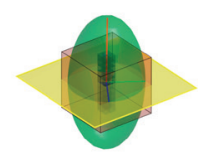

(l)

Fig. 3. Images (a)-(f) represent the constructed benchmarks, and images (g)-(l) represent the respective ellipsoid fitting

with Eq. (15), and verify if the obtained parameters reflect correctly the benchmarks morphology, which was confirmed.

\subsection{Mechanical simulation}

Using the developed homogenization technique, it was possible to define the mechanical properties of homogeneous RVEs from the heterogeneous RVE (characterizing real trabecular bone morphology). With this, and using structural mechanical simulations, it was created a set of models that allow to analyze if the mechanical behavior of the homogenized RVEs is equivalent to the heterogeneous RVEs. Thus, 5 different geometrical models were constructed considering the trabecular bone morphology Figs. 4 b-f, and 5 equivalent homogenized RVEs, represented in Fig. 4g as an example (all homogenized RVEs are simple cubes discretized regularly as the one in Fig. 4g. These mechanical simulations were performed using ABAQUS Unified FEA - SIMULIA ${ }^{\mathrm{TM}} 6.14$ by Dassault Systèmes ${ }^{\circledR}$.

The heterogeneous models were constructed using the same models to which the developed homogeniza- tion technique was applied. The images used to create these models were obtained using micro-computerized tomography (micro-CT) from a foot cuboid bone. Since these images were obtained using micro-CT, they possess a sufficiently high resolution to represent the trabecular structure, but also possess information about the pixel and voxels dimensions, allowing to define, for each voxel a mesh element. Using the same threshold method and parameters used in the homogenization technique, each voxel corresponds to a hexahedric element, which could be defined as bone material or void space. The mechanical properties applied to the elements defined as bone and void in the heterogeneous models are represented in Table 2. It is noteworthy that the geometrical information from the micro-CT imposed the existence of void space in between the trabeculae, making it necessary to define it as a solid "material". Thus, the solution was to define it as a soft material that would not (significantly) interfere with the global structural response of the RVE. Thus, for the "void space", a Young's modulus with a much lower magnitude than the one of the trabecula was attributed. Concerning the boundary condition, two sets of boundary conditions were considered, resulting in two simulation sets. Figure 4 a represents 


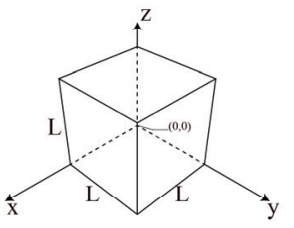

(a)

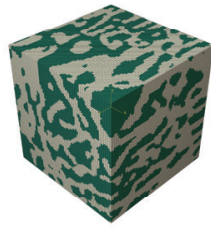

(b)

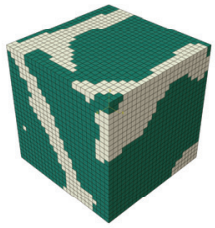

(c)

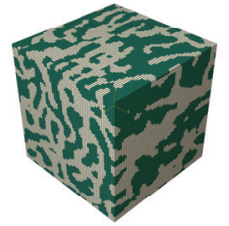

(d)

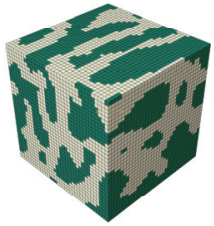

(e)
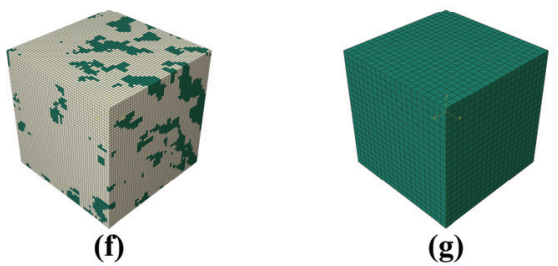

Fig. 4. (a) Geometrical models schematics, (b) heterogeneous model 1, (c) heterogeneous model 2, (d) heterogeneous model 3, (e) heterogeneous model 4,

(f) heterogeneous model 5, (g) generic homogeneous model

a generalization of the geometrical models used in all simulations. For the first set of boundary conditions, at the top layer nodes $(z=L)$ a displacement of $(\bar{w}=0.1 \times L)$ and a displacement restriction $\bar{u}=$ $0 \wedge \bar{v}=0$ were imposed. Also, at the bottom layer nodes $(z=0)$, a displacement restriction $\bar{w}=0$ was applied. For the second set of boundary conditions, at the top layer nodes $(z=L)$ a displacement of $(\bar{w}=0.1 \times L)$ and a displacement restriction $\bar{u}=0 \wedge \bar{v}=0$ were also imposed. However, at the bottom layer nodes $(z=0)$ a complete displacement restriction, $\bar{u}=0 \wedge \bar{v}$ $=0 \wedge \bar{w}=0$ was applied, being $\{u, w, w\}$ the displacement components along the $\{O x, O y, O z\}$ axis, respectively.

Table 2. Mechanical properties used to define the bone by Hong et al. [11] and void space in the heterogeneous models

\begin{tabular}{|l|c|c|}
\hline \multicolumn{3}{|c|}{ Mechanical Properties } \\
\hline & trabecula & void space \\
\hline Young modulus [GPa] & 3.470 & $1.0 \times 10^{-7}$ \\
\hline Poisson's ratio & 0.36 & 0.1 \\
\hline
\end{tabular}

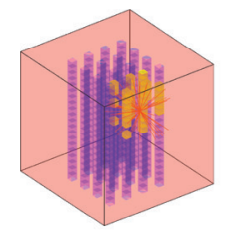

(a)

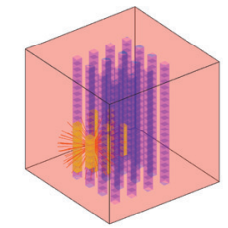

(b)

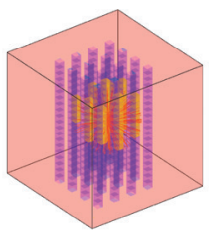

(c)

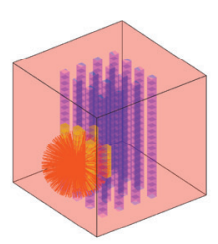

(d)

\section{Results}

This section presents the results obtained from the computational analyses performed to evaluate the behavior of the 3D MIL and also the results obtained from the mechanical simulations, where the mechanical properties of the homogeneous models were defined using the developed homogenization methodology.

\subsection{Validation of MIL method}

Using the created benchmarks, the number of vectors (in the vector list inside the search sphere) that provided stable results with the developed method were analyzed. The behavior of theta, $\theta$, and azimuth, $\omega$ (Fig. 6a), and the length of the radius that defines the fitted ellipsoid (Fig. 6b) were analyzed. The results in Figs. 6a) and 6b) were obtained applying the proposed method to the benchmarks pres-

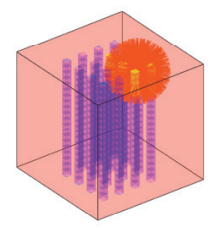

(e)

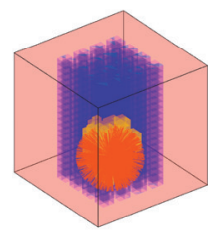

(f)

Fig. 5. Different number of vectors inside the search sphere:

(a) 50 vectors, (b) 100 vectors, (c) 250 vectors, (d) 500 vectors, (e) 1000 vectors and (f) 1500 vectors 


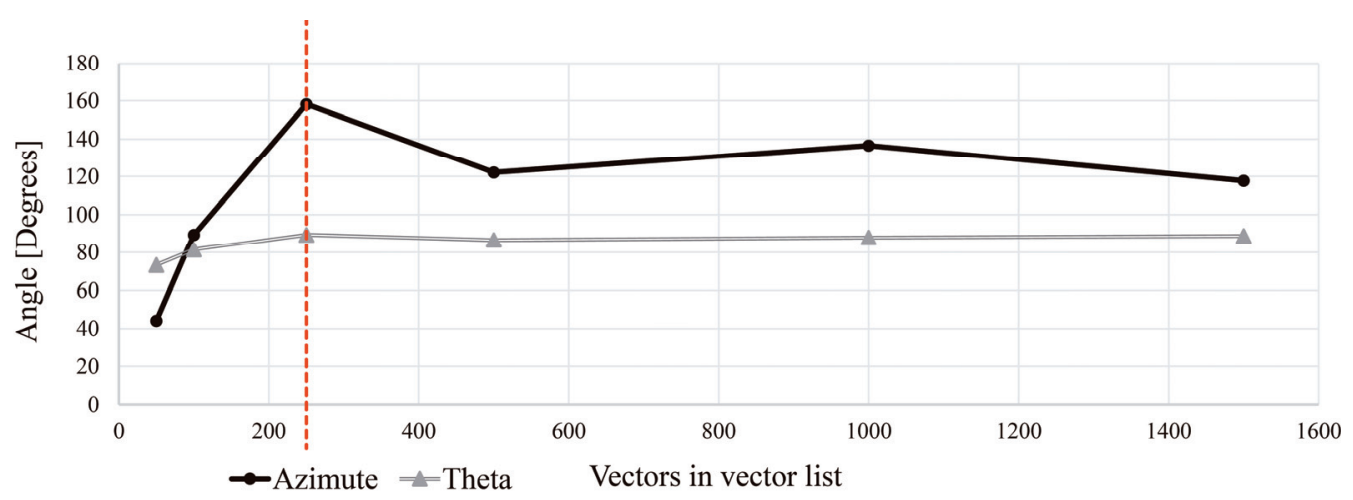

(a)

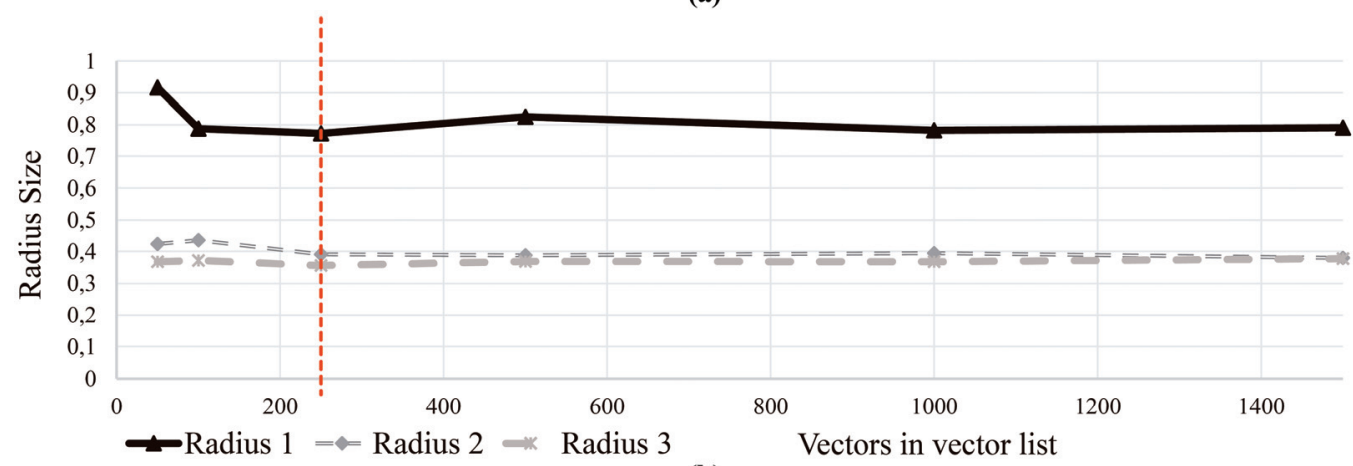

(b)

Fig. 6. (a) Evolution of the obtained $\omega$ and $\theta$ with the increase of the number of vectors in the vector list inside the search sphere, (b) Evolution of the obtained $r_{1}, r_{2}$ and $r_{3}$ with the increase of the number of vectors in the vector list inside the search sphere

Table 3. Parameters obtained using the developed method, defining the local mechanical properties of the constructed benchmarks

\begin{tabular}{|c|c|c|c|c|c|c|}
\hline \multicolumn{7}{|c|}{ Benchmark } \\
\hline & 1 & 2 & 3 & 4 & 5 & 6 \\
\hline$\omega$ & 11.985 & 0.219 & 43.701 & 44.373 & 28.517 & 26.107 \\
\hline$\theta$ & 89.546 & 0.089 & 34.733 & 35.731 & 89.654 & 89.138 \\
\hline$E_{1}$ & 333.440 & 333.440 & 15.509 & 111.540 & 15.509 & 66.301 \\
\hline$E_{2}$ & 253.905 & 249.008 & 2.133 & 55.018 & 5.280 & 34.569 \\
\hline$E_{3}$ & 247.595 & 246.993 & 2.085 & 54.725 & 5.176 & 34.354 \\
\hline$G_{1}$ & 159.084 & 159.084 & 7.731 & 54.720 & 7.731 & 32.757 \\
\hline$G_{2}$ & 95.211 & 93.374 & 0.993 & 23.472 & 2.459 & 15.350 \\
\hline$G_{3}$ & 103.035 & 102.784 & 0.958 & 24.360 & 2.379 & 15.554 \\
\hline$v_{1}$ & 0.048 & 0.048 & 0.003 & 0.019 & 0.003 & 0.012 \\
\hline$v_{2}$ & 0.333 & 0.333 & 0.074 & 0.172 & 0.074 & 0.126 \\
\hline$v_{3}$ & 0.202 & 0.202 & 0.088 & 0.123 & 0.088 & 0.104 \\
\hline$\rho_{\text {app }}$ & 0.336 & 0.336 & 0.021 & 0.134 & 0.021 & 0.084 \\
\hline
\end{tabular}

ent in Fig. 5. In Table 3, the several parameters obtained using the proposed method for all benchmarks of Fig. 3 are shown.

\subsection{Mechanical simulation}

The performed mechanical simulations aimed to evaluate whether the developed homogenization tech- nique was able to define the mechanical properties of the heterogeneous domains, enabling to define homogeneous models (mechanically equivalent to the trabecular heterogeneous models defined by the heterogeneous geometrical models). For the constructed models, both the von Mises stress field and the displacement field were obtained, Figs. 7 and 8 , respectively. Figure 7 regards the first set of boundary conditions applied to the models. In each sub-image 

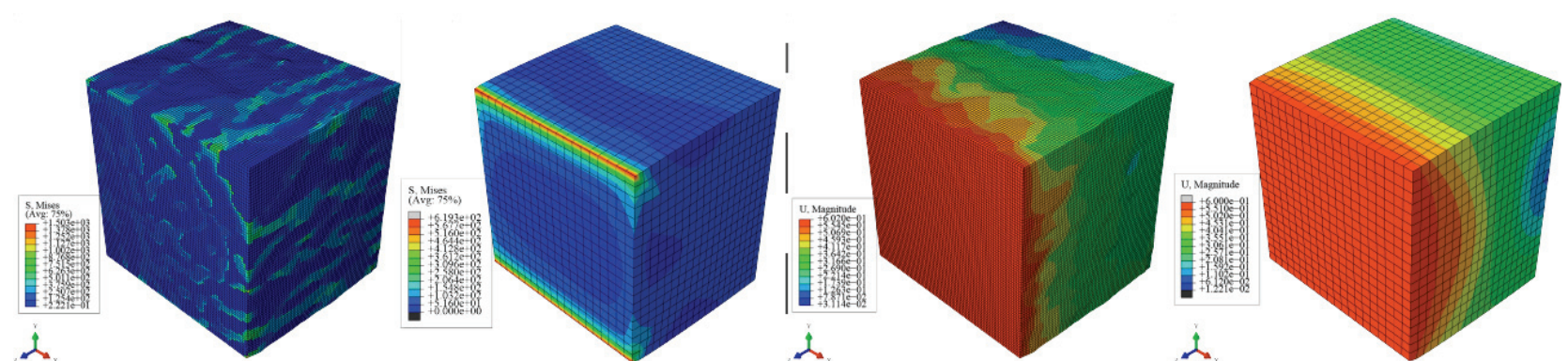
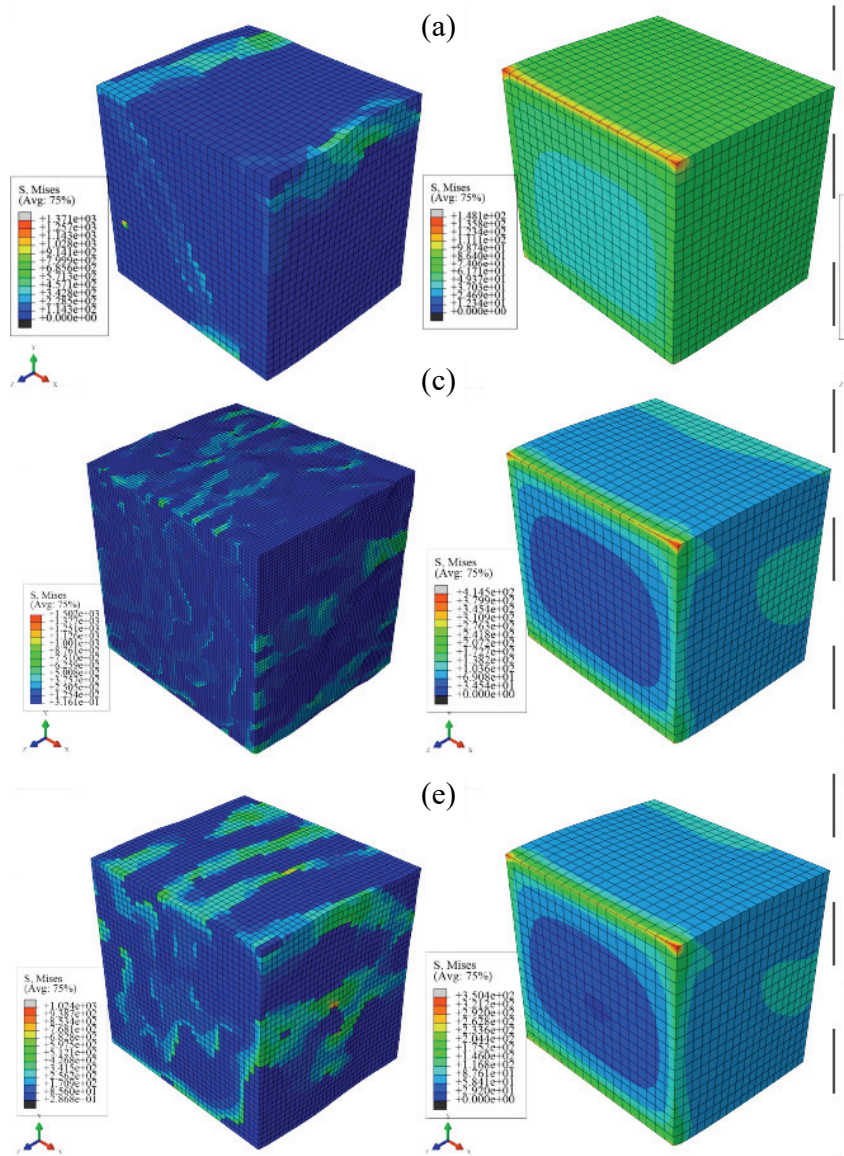

(g)

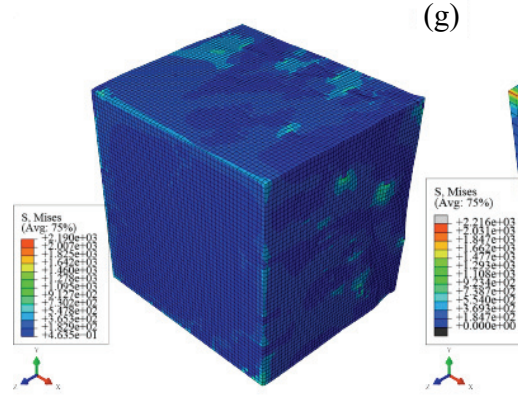

(i)
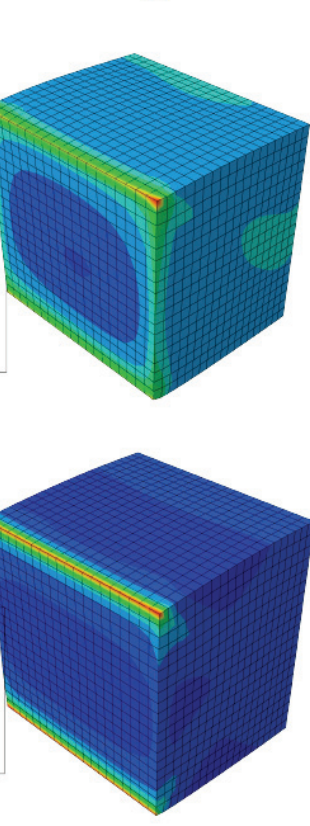

$1+$

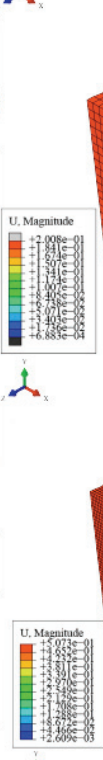

i.

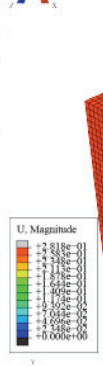

$\dot{2}$
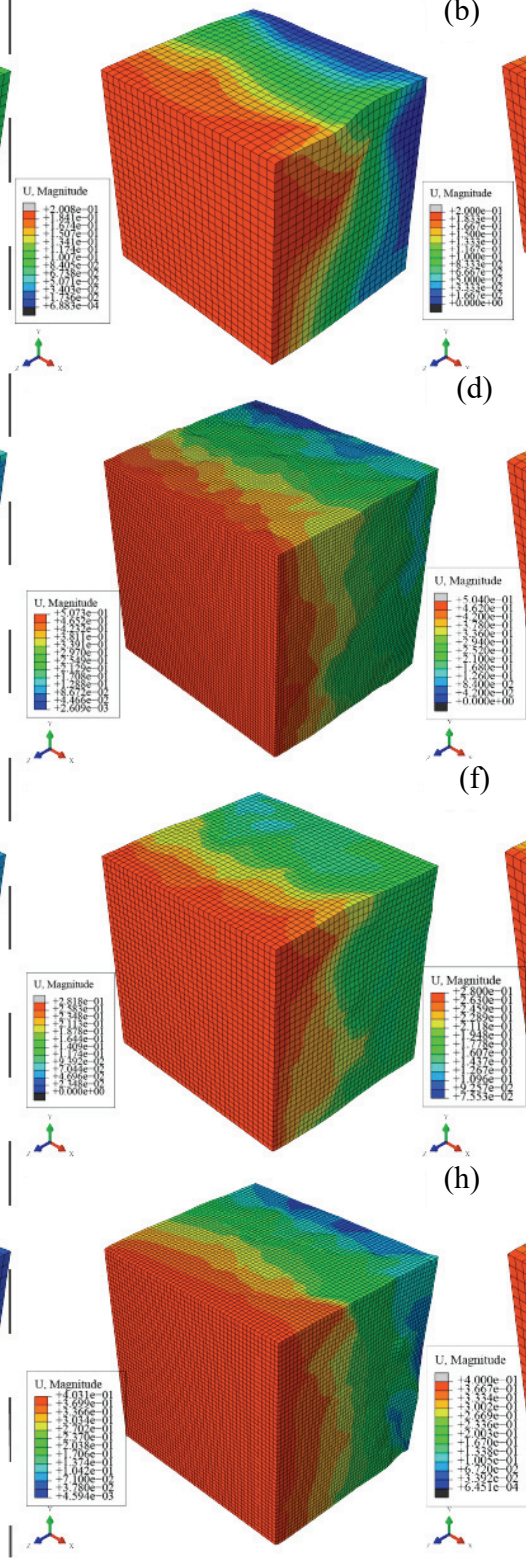

(b)

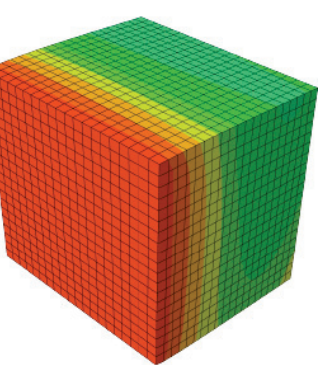

(d)

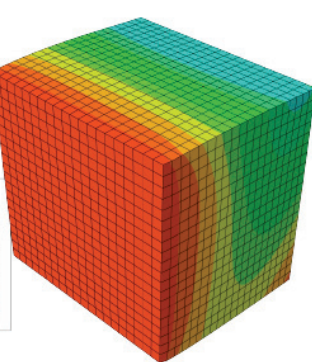

(f)

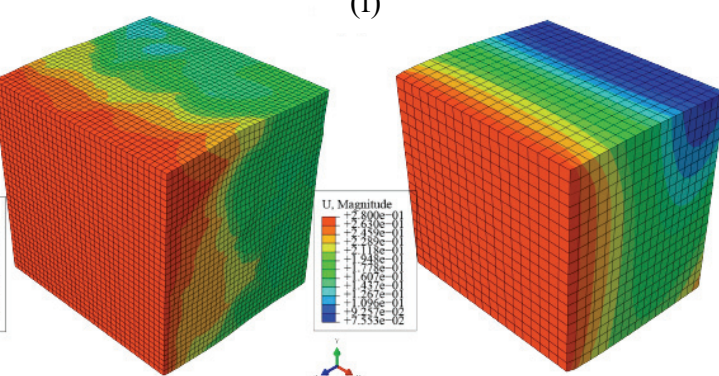

(h)

(j)

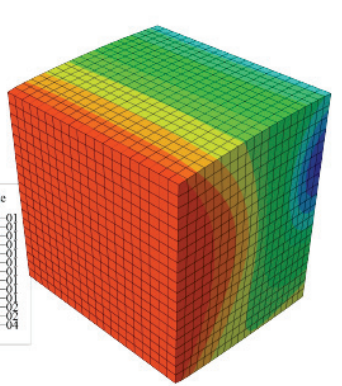

Fig. 7. Von Mises stress and displacement for the mechanical simulations using the first set of boundary conditions. (a), (c), (e), (g), (i): Modelss 1 to 5 - heterogeneous and equivalent homogeneous model von Mises stress; (b), (d), (f), (h) (j): Models 1 to 5 - heterogeneous and equivalent homogeneous model displacement

of this figure, the result for the heterogeneous model and its homogeneous equivalent are presented. Figure 8 regards the second set of boundary conditions.
Also, in this figure, each sub-image shows the result for the heterogeneous model and its homogeneous equivalent. 

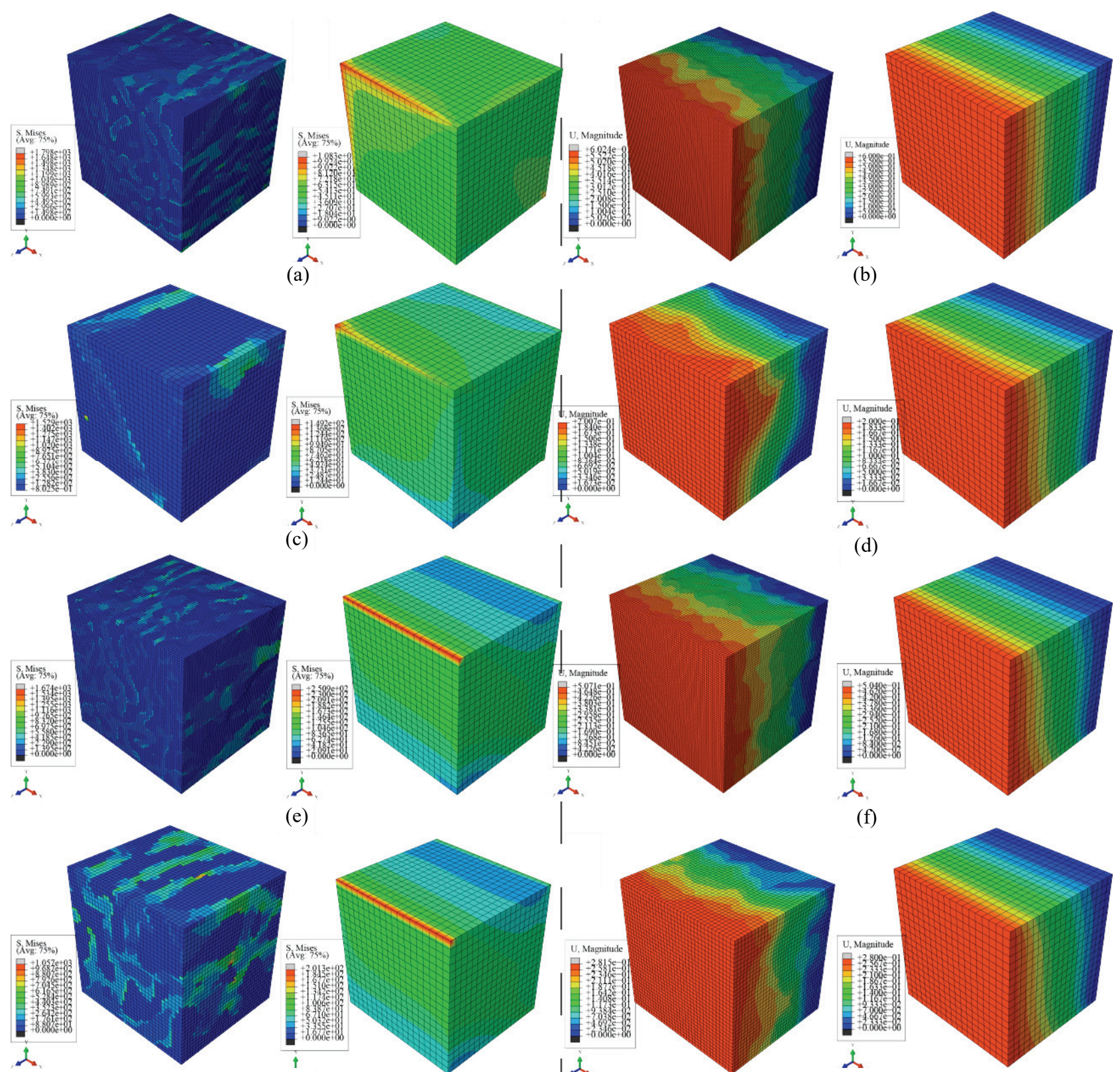

(e)

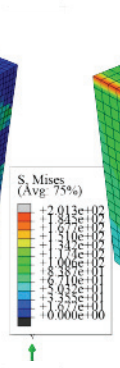

(g)

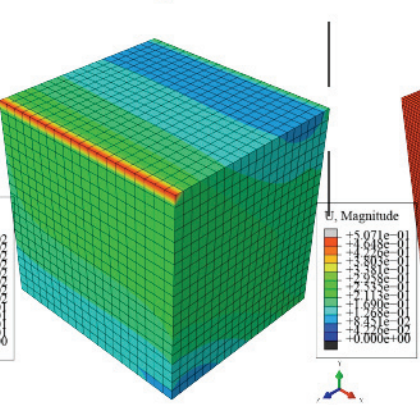

$\stackrel{1}{1}$
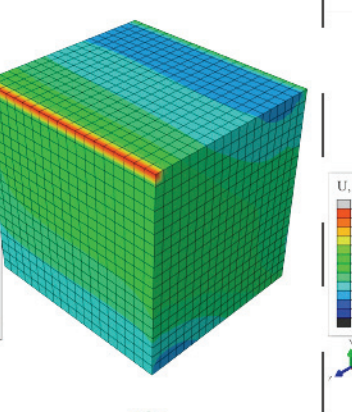

(f)
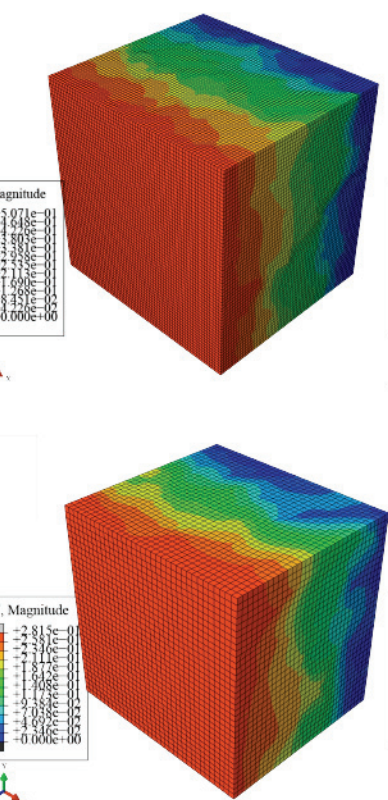

(h)
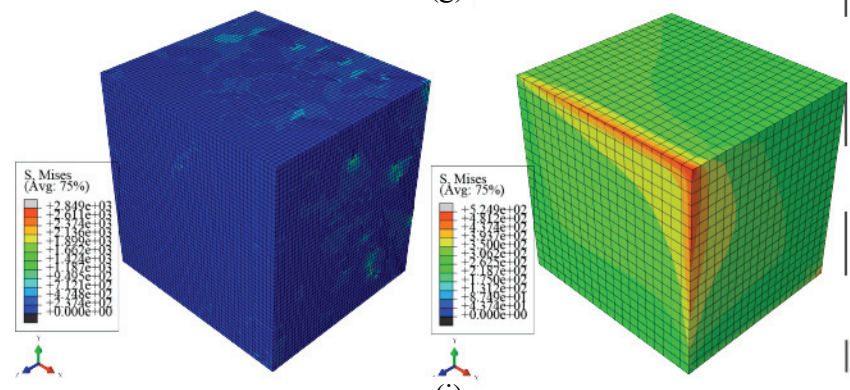

(i)

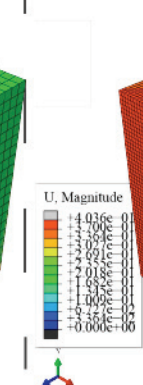

人.
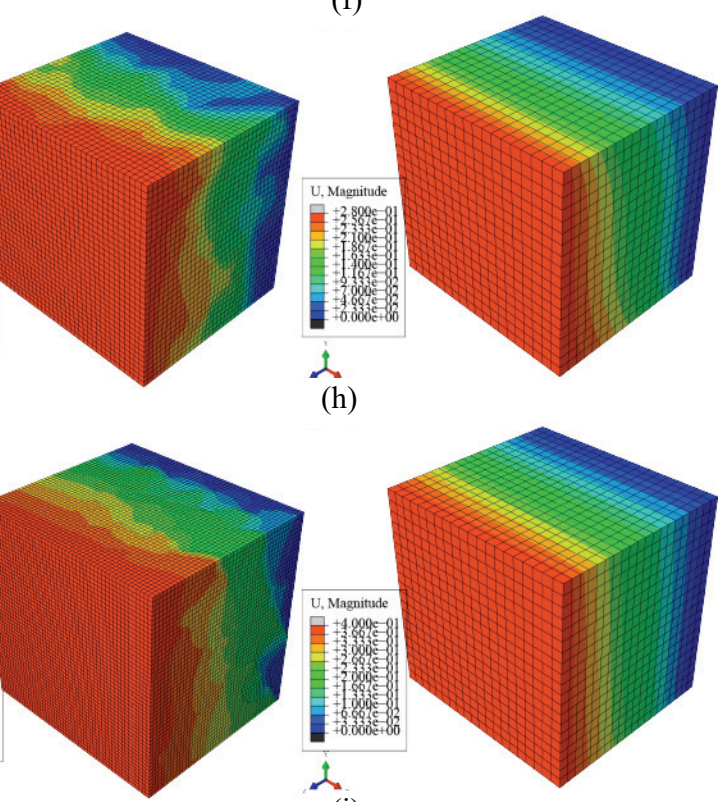

(j)

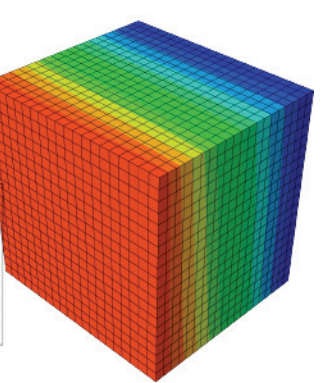

Fig. 8. Von Mises stress and displacement for the mechanical simulations using the second set of boundary conditions.

(a), (c), (e), (g), (i): Models 1 to 5 - heterogeneous and equivalent homogeneous model von Mises stress; (b), (d), (f), (h), (j): Models 1 to 5 - heterogeneous and equivalent homogeneous model displacements 


\section{Discussion}

This section presents discussion regarding the results obtained from the tests performed to evaluate the behavior of the 3D MIL. Moreover, the results obtained from the mechanical simulations, where the mechanical properties of the homogeneous models were defined using the developed homogenization methodology, are also analyzed and discussed.

From the results obtained using the benchmarks, concerning the behavior of theta, $\theta$, and azimuth, $\omega$ (Fig. 6a), and the length of the radius that defines the fitted ellipsoid (Fig. 6b), it is possible to visualize that starting from 250 vectors, highlighted with vertical dotted line, the results stabilize, which means that the convergence is achieved and using a higher number of vectors does not increases significantly the accuracy of the solution. Thus, using 250 vectors enables us to obtain results very close to the ones obtained considering 1500 vectors. The advantage is the computational time, an analysis with 250 vectors is much faster than an analysis with 1500 vectors. Concerning the values from Table 3, when comparing the results of benchmark 1 and 2, Figs. 3a and b, respectively, and considering the benchmarks characteristic, it is possible to conclude that these results are as expected. Benchmarks 1 and 2 have the same number of bone voxels, reflecting the high similarity between $E_{1}, E_{2}, E_{3}, G_{1}$, $G_{2}, G_{3}, v_{1}, v_{2}, v_{3}$ and $\rho_{a p p}$, however, when considering $\theta$ and $\omega$, these two parameters reflect the different benchmark principal direction.

Concerning benchmark 3 and 4 (Figs. 3c, d) that, as previously described, have an oblique principal direction, $\theta$ and $\omega$ parameters reflect this oblique topology. The remain parameters, $E_{1}, E_{2}, E_{3}, G_{1}, G_{2}, G_{3}, v_{1}, v_{2}$, $v_{3}$ and $\rho_{a p p}$, reflect the different number of voxels that define the thickness of the bone region. Comparing the results obtained with benchmark 5 and benchmark 6 (Figs. 3e, f), the values in Table 3 make it possible to understand that $\theta$ and $\omega$ parameters are highly similar. The remaining parameters, $E_{1}, E_{2}, E_{3}, G_{1}, G_{2}$, $G_{3}, v_{1}, v_{2}, v_{3}$ and $\rho_{a p p}$ are not comparable. These results were the expected ones, because, as previously described, these benchmarks have the same material principal direction but a different number of bone voxels. Although insignificant, the difference between values (that should be exactly the same, as for example $E_{3}$ ) for benchmark 1 and 2 occurs because of the random selection of the centroid of the search sphere. In Figures $3 \mathrm{~g}$ to 1 , the fitted ellipsoids are presented. In these images it is visible that the fitted ellipsoid, by visual inspection, has the same material principal direction as the fabricated benchmarks, reenforcing the hypothesis that the developed methodology can define and encode trabecular bone orientation and anisotropy.

Concerning the mechanical simulation results, it was observed for all the heterogeneous models results that bone zones, which define the trabeculae, present higher values of the stress. For the case of the displacement values, as expected, higher values are observed in the region close to $z=L$, which is the location were the imposed displacement was applied. As expected, a gradual apparent linear decrease of the displacement from $z=L$ to $z=0$ is also observed. Concerning the homogeneous models, they present, when compared with the heterogeneous models, a smoother von Mises stress spatial distributions, reflecting the homogeneous nature of the model. Analyzing the displacement values, the images show that, similarly to the heterogeneous models, the higher displacement values appear in the nodes where the enforced displacement was applied (the region close to $z=L$ ), and that a gradual decrease of the displacement values down to $z=0$ occurs. As the geometrical nature of the models is so different, it is difficult to compare these models using a local perspective of the models. Thus, to evaluate whether the homogeneous models have an equivalent behavior to the heterogeneous model, a relation between the sum of the $z$ components of the reaction forces, at $z=0$, $\sum R_{z}^{z=0}$, and the displacement applied at $z=L$, $u_{3}^{z=L}$, Eq. (16) was defined, leading to a homogenized value of stiffness $K$.

$$
K=\frac{\sum R_{z}^{z=0}}{u_{3}^{z=L}}
$$

The results obtained using Eq. (16) are represented in Figs. 9 and 10. Regarding the results of Fig. 9, concerning the first boundary conditions set, the difference between the homogeneous and the heterogeneous model is of $56 \%$ for the model $1,0 \%$ - for model 2 , $26 \%$ - for model $3,38 \%$ - for model 4 and $9 \%$ - for model 5, representing an overall average difference of $21 \%$. For the case of mechanical simulations performed, using the second set of boundary conditions, the difference between the homogeneous and the heterogeneous model is of $6 \%$ for the model $1,7 \%$ - for model 2, $11 \%$ - for model 3, 3\% - for model 4 and 59\% - for model 5 , representing an overall average difference of $14 \%$. 


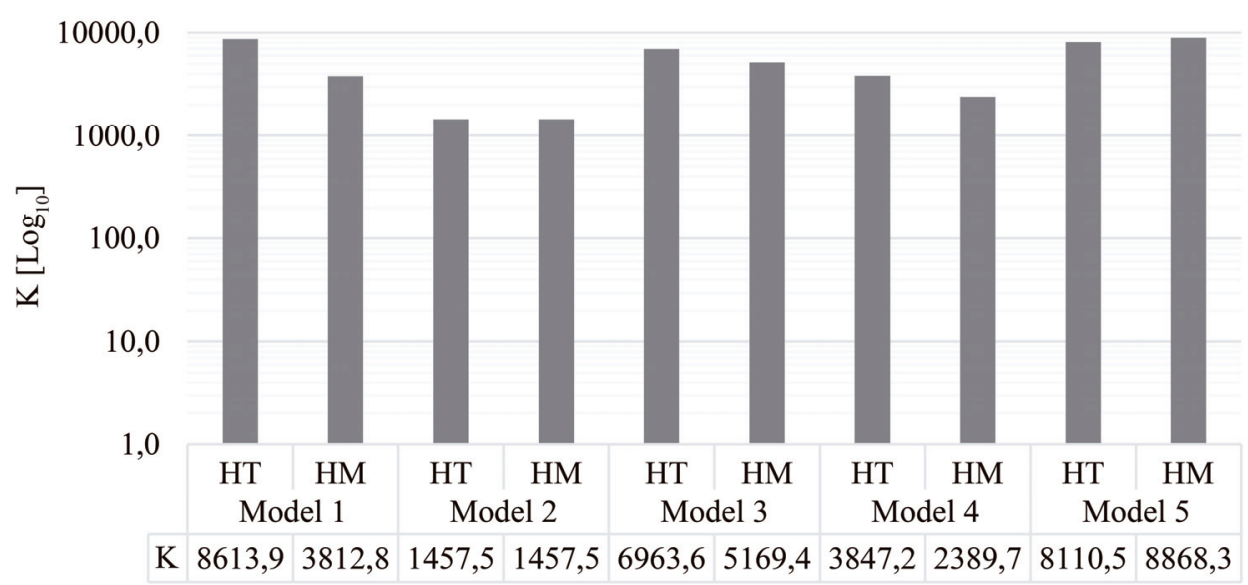

Fig. 9. Homogenized stiffness $K$ for the mechanical simulation results performed using the first set of boundary conditions,

for the homogeneous (HM) and heterogeneous (HT) models

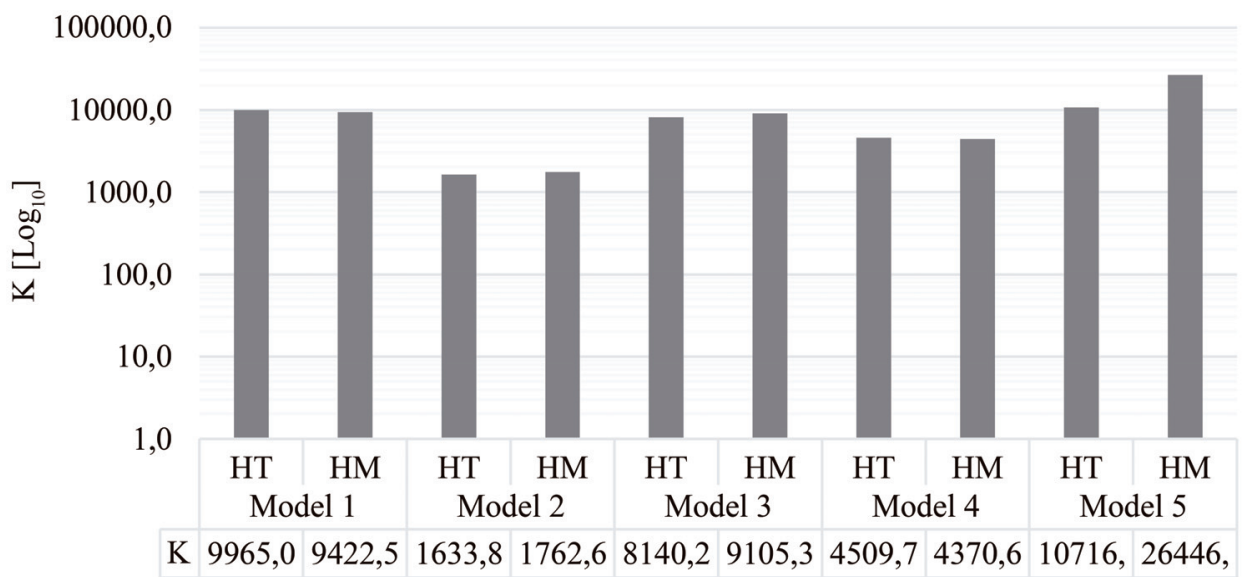

Fig. 10. Homogenized stiffness $K$ for the mechanical simulation results performed using the second set of boundary conditions, for the homogeneous (HM) and heterogeneous (HT) models

10000.00

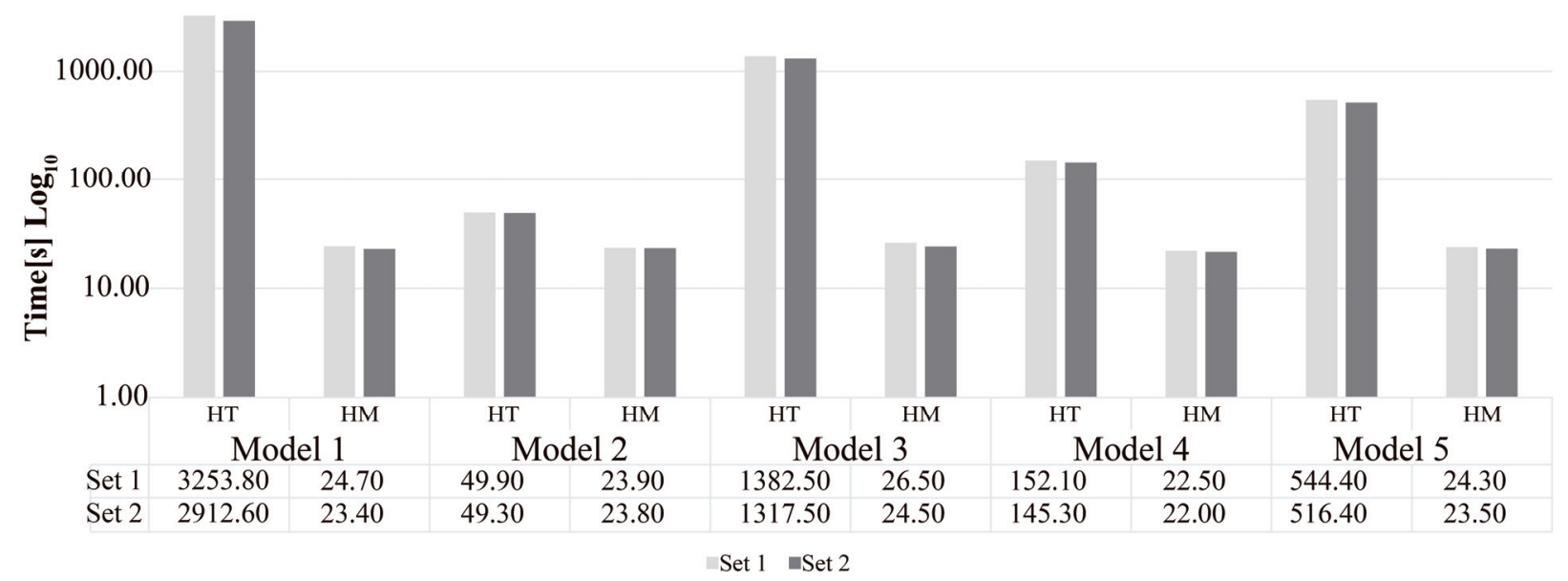

Fig. 11. Computational cost of the mechanical simulations performed using the 6 heterogeneous models (HT), and its 6 equivalent homogeneous models (HM), for the two sets of boundary conditions, set 1 and set 2 
One factor possessing a significant impact in multiscale simulation is the computational cost. Thus, the computation cost of the developed method was analyzed. In Figure 11, the computational cost of the performed mechanical simulation for each model, and for both boundary condition sets (set 1 and set 2) is presented. The developed method leads to a maximum decrease of $99.2 \%$ of the computation time, in the case of the model 1 , a minimum of $51.7 \%$ for model 4 and a $90.3 \%$ average decrease of the computational time of all the models.

\section{Conclusions}

In this work, a method to define the homogeneous equivalent mechanical properties of the highly heterogeneous trabecular bone tissue was proposed. The method uses the fabric tensor concept and two material laws [1], [4], [26]. Regarding the fabric tensor, the results show that if actually encodes the trabecular bone orientation and anisotropy. Also, using the set of fabricated benchmarks, the robustness of the method was evaluated, which made it possible to conclude that it provides reliable information about the principal directions and anisotropy. The considered material laws (Belinha's law and Agic's law) complemented the fabric tensor information, by providing the Young's modulus of the homogenized material in the material's principal direction and the Poisson's coefficient (by using the average apparent density of the complete domain). The mechanical simulations analyzed heterogeneous and homogeneous models with equivalent mechanical properties obtained using the developed method. The simulations shown that the developed method properly defines the equivalent mechanical proprieties of the homogeneous models. Since the analyzed geometric models have a distinct discretization (the nodes of two distinct models do not spatially coincide), it was not possible to compare the results using a local nodal comparison, in which the displacements and stresses at the same nodal coordinates in both models are analyzed. Thus, the concept of homogenized stiffness $K$ was considered, proving to be an efficient tool to compare the homogeneous and heterogeneous models. Considering the differences in the homogenized stiffness $K$ between the heterogeneous and the equivalent homogeneous models and the differences between the computation cost, it is possible to conclude that the proposed homogenization technique is capable to deliver accurate homogenized mechanical properties to be applied to very coarse homogenized discrete models in a fraction of time. Thus, the proposed homogeni- zation method possesses the potential to significantly decrease the computational cost of demanding multiscale analyses without compromising its accuracy.

\section{Acknowledgements}

The authors truly acknowledge the funding provided by Ministério da Ciência, Tecnologia e Ensino Superior - Fundação para a Ciência e a Tecnologia (Portugal), under Grants SFRH/BD/110047/2015, and by project funding MIT-EXPL/ISF/0084/2017 and UIDB/50022/2020.

\section{References}

[1] Agić A., Nikolić V., MiJović B., The cancellous bone multiscale morphology-elasticity relationship, Collegium Antropologicum, 2006, 30 (2), 409-414. http://www.ncbi.nlm.nih.gov/ pubmed/16848160

[2] Belinha J., Meshless Methods in Biomechanics, Vol. 16. V.E. Brimkov, R.P. Barneva (Eds.), Springer International Publishing; Cham 2014 (Lecture Notes in Computational Vision and Biomechanics), DOI: 10.1007/978-3-319-06400-0.

[3] Belinha J., Dinis L.M.J.S.M.J.S., NATAl Jorge R.M.M., The analysis of the bone remodelling around femoral stems: A meshless approach, Mathematics and Computers in Simulation, 2016, 121, 64-94, http://www.sciencedirect. com/science/article/pii/S0378475415001895. DOI: 10.1016/ j.matcom.2015.09.002.

[4] Belinha J., Jorge R.M.N., Dinis L.M.J.S., A meshless microscale bone tissue trabecular remodelling analysis considering a new anisotropic bone tissue material law, Computer Methods in Biomechanics and Biomedical Engineering, 2013, 16 (11), 1170-1184, DOI: 10.1080/ 10255842.2012.654783.

[5] Cai X., Peralta L., Brenner R., Iori G., Cassereau D., Raum K., Laugier P., Grimal Q., Anisotropic elastic properties of human cortical bone tissue inferred from inverse homogenization and resonant ultrasound spectroscopy, Materialia, 2020, 11, 100730. https://www.sciencedirect.com/ science/article/pii/S2589152920301472. DOI: 10.1016/ J.MTLA.2020.100730.

[6] CARTer D.R., Fyhrie D.P., Whalen R.T., Trabecular bone density and loading history: Regulation of connective tissue biology by mechanical energy, Journal of Biomechanics, 1987, 20 (8), 785-794, DOI: 10.1016/0021-9290(87)90058-3.

[7] Carter D.R., Van Der Meulen M.C., Beaupré G.S., Mechanical factors in bone growth and development, Bone, 1996, 18 (1 Suppl.), 5S-10S, DOI: 10.1016/8756-3282(95)00373-8.

[8] CowIN S.C., The relationship between the elasticity tensor and the fabric tensor, Mechanics of Materials, 1985, 4 (2), 137-147, DOI: 10.1016/0167-6636(85)90012-2.

[9] Fitzgibbon A.W., Pilu M., Fisher R.B., Direct least squares fitting of ellipses, Proceedings of 13th International Conference on Pattern Recognition. IEEE; 1996, Vol. 1, 253-257, www. dai.ed.ac.ukgroupssmvuuellipse-demo.html, DOI: 10.1109/ ICPR.1996.546029.

[10] Goda I., Assidi M., Belouettar S., Ganghoffer J.F., A micropolar anisotropic constitutive model of cancellous bone from discrete homogenization, Journal of the Mechanical Behavior of Biomedical Materials, 2012, 16, 87-108. 
https://www.sciencedirect.com/science/article/pii/S17516161 12002135, DOI: 10.1016/J.JMBBM.2012.07.012.

[11] Hong J., Cha H., PARK Y., Lee S., Khang G., Kim Y., Elastic Moduli and Poisson's Ratios of Microscopic Human Femoral Trabeculae, 11th Mediterranean Conference on Medical and Biomedical Engineering and Computing 2007. Berlin, Heidelberg: Springer Berlin Heidelberg, 2007, 274-277, DOI: 10.1007/978-3-540-73044-6_68.

[12] Kersh M.E., Zysset P.K., PAHR D.H., Wolfram U., LARSSON D., PANDY M.G., Measurement of structural anisotropy in femoral trabecular bone using clinical-resolution $C T$ images, Journal of Biomechanics, 2013, 46 (15), 2659-2666, DOI: 10.1016/j.jbiomech.2013.07.047.

[13] LANDIS W.J., The strength of a calcified tissue depends in part on the molecular structure and organization of its constituent mineral crystals in their organic matrix, Bone, 1995, 16 (5), 533-544, DOI: 10.1016/8756-3282(95)00076-P.

[14] Marco M., Giner E., Caeiro-Rey J.R., Miguélez M.H., LARRAÍNZAR-GARIJO R., Numerical modelling of hip fracture patterns in human femur, Computer Methods and Programs in Biomedicine, 2019, 173, 67-75, https:// www.sciencedirect.com/science/article/pii/S0169260719302275, DOI: 10.1016/J.CMPB.2019.03.010.

[15] Martínez-Reina J., Domínguez J., García-Aznar J.M., Effect of porosity and mineral content on the elastic constants of cortical bone: a multiscale approach, Biomechanics and Modeling in Mechanobiology, 2011, 10 (3), 309-322, http://link.springer.com/10.1007/s10237-010-0236-4. DOI: 10.1007/s10237-010-0236-4.

[16] Moreno R., Borga M., SMEDBy O., Techniques for Computing Fabric Tensors - A Review, Mathematics and Visualization, 2014, Vol. 5, 271-292, DOI: 10.1007/978-3-642-54301-2_12.

[17] Moreno R., SMedBy Ö., PAHR D.H., Prediction of apparent trabecular bone stiffness through fourth-order fabric tensors, Biomechanics and Modeling in Mechanobiology, 2016, 15 (4), 831-844, http://link.springer.com/10.1007/s10237-015-0726-5, DOI: $10.1007 / \mathrm{s} 10237-015-0726-5$.

[18] NAZemi S.M., CoOper D.M.L., Johnston J.D., Quantifying trabecular bone material anisotropy and orientation using low resolution clinical CT images: A feasibility study, Medical Engineering and Physics, 2016, 38 (9), 978-987, https://
www.sciencedirect.com/science/article/pii/S1350453316301254, DOI: 10.1016/J.MEDENGPHY.2016.06.011.

[19] PERrin E., Bou-SAÏD B., MAssi F., Numerical modeling of bone as a multiscale poroelastic material by the homogenization technique, Journal of the Mechanical Behavior of Biomedical Materials, 2019, 91, 373-382, https:// www.sciencedirect.com/science/article/pii/S1751616118309020, DOI: 10.1016/J.JMBBM.2018.12.015.

[20] Peyroteo M.M.A., Belinha J., Vinga S., Dinis L.M.J.S., NATAL JoRge R.M., Mechanical bone remodelling: Comparative study of distinct numerical approaches, Engineering Analysis with Boundary Elements, 2019, 100, 125-139, https:// www.sciencedirect.com/science/article/pii/S0955799717304022 ?via\%3Dihub, DOI: 10.1016/j.enganabound.2018.01.011.

[21] Rho J.-Y., Kuhn-Spearing L., Zioupos P., Mechanical properties and the hierarchical structure of bone, Medical Engineering and Physics, 1998, 20 (2), 92-102, DOI: 10.1016/ S1350-4533(98)00007-1.

[22] Sabet F.A., Raeisi Najafi A., Hamed E., Jasiuk I., Modelling of bone fracture and strength at different length scales: a review, Interface Focus, 2016, 6 (1), 20150055, DOI: 10.1098/rsfs.2015.0055.

[23] SANZ-Herrera J.A.A., García-AZnar J.M.M., Doblaré M., On scaffold designing for bone regeneration: A computational multiscale approach, Acta Biomaterialia, 2009, 5 (1), 219-229, https://www.sciencedirect.com/science/article/pii/ S1742706108002286, DOI: 10.1016/J.ACTBIO.2008.06.021.

[24] Whitehouse W.J., The quantitative morphology of anisotropic trabecular bone, Journal of Microscopy, 1974, 101 (2), 153-168, DOI: 10.1111/j.1365-2818.1974.tb03878.x.

[25] WNEK G.E., Bowlin G.L., Encyclopedia of biomaterials and biomedical engineering, 2nd ed., CRC Press, New York, 2004, https://www.crcpress.com/Encyclopedia-of-Biomaterials-andBiomedical-Engineering/Wnek-Bowlin/p/book/9781420078022, DOI: $10.5860 /$ choice.42-1940.

[26] WolfF J., The Law of Bone Remodelling, Springer, Berlin, Heidelberg, 1886, http://linkinghub.elsevier.com/retrieve/pii/ 8756328295003738, DOI: 10.1007/978-3-642-71031-5.

[27] ZiEnkiewicz O., TAYLOR R., ZhU J.Z., The Finite Element Method: Its Basis and Fundamentals, 7th ed., ButterworthHeinemann, 2013. 